\title{
Fundamental structure of Fresnel diffraction: longitudinal uniformity with respect to fractional Fourier order
}

\author{
Haldun M. Ozaktas, ${ }^{*}$ Sercan Ö. Arık, and Türker Coşkun \\ Bilkent University, Department of Electrical Engineering TR-0680o Bilkent, Ankara, Turkey \\ ${ }^{*}$ Corresponding author: haldun@ee.bilkent.edu.tr
}

Received September 15, 2011; revised November 17, 2011; accepted November 18, 2011; posted November 18, 2011 (Doc. ID 154665); published December 24, 2011

\begin{abstract}
Fresnel integrals corresponding to different distances can be interpreted as scaled fractional Fourier transformations observed on spherical reference surfaces. Transverse samples can be taken on these surfaces with separation that increases with propagation distance. Here, we are concerned with the separation of the spherical reference surfaces along the longitudinal direction. We show that these surfaces should be equally spaced with respect to the fractional Fourier transform order, rather than being equally spaced with respect to the distance of propagation along the optical axis. The spacing should be of the order of the reciprocal of the space-bandwidth product of the signals. The space-dependent longitudinal and transverse spacings define a grid that reflects the structure of Fresnel diffraction. @ 2011 Optical Society of America

OCIS codes: $\quad 070.2575,070.2580,070.7345,050.1940,050.5082,070.0070$.
\end{abstract}

Fresnel integrals can be interpreted as scaled fractional Fourier transformations observed on spherical reference surfaces. Earlier, we showed that by appropriately choosing sample points on these reference surfaces, it is possible to represent the diffracted signals in a nonredundant manner [1]. Here we show that these reference surfaces should be spaced equally with respect to the fractional Fourier transform order, rather than with respect to the distance of propagation.

We introduce dimensionless coordinates [2] and work with a single transverse dimension. Let $\hat{f}(x)$ and $\hat{F}\left(\sigma_{x}\right)$ denote the space and frequency representation of a signal. We use $f(u)$ and $F(\mu)$ to denote corresponding functions with dimensionless arguments $u$ and $\mu$ as follows: $\hat{f}(x) \equiv s^{-1 / 2} f(x / s), \hat{F}\left(\sigma_{x}\right) \equiv s^{1 / 2} F\left(s \sigma_{x}\right)$, where $s$ is a scaling parameter.

The fractional Fourier transform (FRT) of a function $f(u)$ is denoted as $f_{a}(u)$, where $a$ is the FRT order [2]. The Fresnel integral describes the propagation of light from one transverse plane along the optical axis to another. The output field $\hat{g}(x, z)$ is related to the input field $\hat{f}(x)$ by $\hat{g}(x, z)=\int_{-\infty}^{\infty} \hat{h}\left(x-x^{\prime}, z\right) \hat{f}\left(x^{\prime}\right) \mathrm{d} x^{\prime}$, where $\hat{h}(x, z)=\exp (i 2 \pi z / \lambda) \exp (-i \pi / 4)(\lambda z)^{-1 / 2} \exp \left(i \pi x^{2} / \lambda z\right)$, where $z$ is the distance of propagation and $\lambda$ is the wavelength.

The two-dimensional (2D) Fourier transform (FT) of $\hat{g}(x, z)$ can be found by first considering the FT with respect to $x$, using the convolution property, and finally transforming with respect to $z$ :

$$
\hat{G}\left(\sigma_{x}, \sigma_{z}\right)=\hat{F}\left(\sigma_{x}\right) \delta\left(\sigma_{z}-\left(1 / \lambda-\lambda \sigma_{x}^{2} / 2\right)\right) .
$$

This function is a modulated impulsive edge along the parabola $\sigma_{z}=1 / \lambda-\lambda \sigma_{x}^{2} / 2$ (the parabola in Fig. 1).

It is known that the Fresnel integral can be decomposed into an FRT followed by magnification followed by chirp multiplication [2-6]

$$
\hat{g}(x, \boldsymbol{z})=e^{i 2 \pi z / \lambda} e^{-i a \pi / 4} \sqrt{\frac{1}{s M}} \exp \left(\frac{i \pi x^{2}}{\lambda R}\right) f_{a}\left(\frac{x}{s M}\right),
$$

where

$$
\begin{aligned}
a & =\frac{2}{\pi} \arctan \frac{\lambda z}{s^{2}}, \quad M=\sqrt{1+\frac{\lambda^{2} z^{2}}{s^{4}}}, \\
R & =\frac{s^{4}+\lambda^{2} z^{2}}{\lambda^{2} z}
\end{aligned}
$$

If we choose to observe the diffracted light on a spherical reference surface of radius $R$, the chirp multiplication can be dispensed with, and we simply observe the FRT of the input, magnified by $M$ [2]. (The constant phase terms $e^{i 2 \pi d / \lambda} e^{-i a \pi / 4}$ are not of significance.) Equation (2) holds true regardless of the choice of $s$.

We assume that the energy of the signal at the $z=0$ plane is confined to an ellipse with diameters $\Delta x$ and $\Delta \sigma_{x}$ in the space-frequency plane, in the sense that most of the signal energy lies within this ellipse. $\Delta x$ and $\Delta \sigma_{x}$ also correspond to the space and frequency extents of the signal. Since a frequency extent of $\Delta \sigma_{x}$ implies a sampling interval of $1 / \Delta \sigma_{x}$, we would need $N=$ $\Delta x /\left(1 / \Delta \sigma_{x}\right)=\Delta x \Delta \sigma_{x}$ samples to characterize the signal in terms of its samples, a quantity also referred to as the space-bandwidth product.

In [1] , we showed that if we choose $s=\sqrt{\Delta x / \Delta \sigma_{x}}$, then the spatial and spatial frequency extents of the diffracted signal on the spherical reference surface are

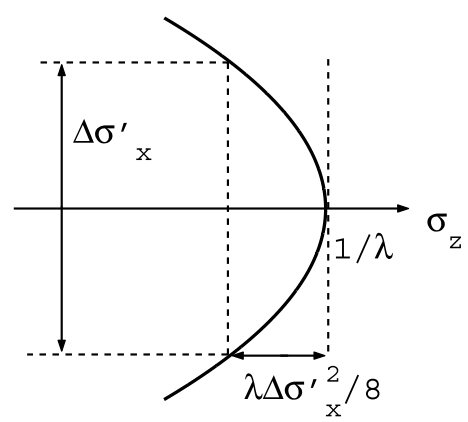

Fig. 1. Truncation of $\hat{G}\left(\sigma_{x}, \sigma_{z}\right)$ in the $\sigma_{z}-\sigma_{x}$ plane. 


$$
\Delta x^{\prime}=M \Delta x, \quad \Delta \sigma_{x}^{\prime}=\Delta \sigma_{x} / M,
$$

respectively. Note that with this choice of $s$, the spacebandwidth product $\Delta x^{\prime} \Delta \sigma_{x}^{\prime}$ on this surface is the same as that on the input plane (other values of $s$ result in larger space-bandwidth products). This implies that the number of samples $\Delta x^{\prime} /\left(1 / \Delta \sigma_{x}^{\prime}\right)$ required to represent the diffracted signal is the same as that required to represent the original signal, but these samples are spaced further apart. In [1] , we further discuss sampling and computation issues [7-16]. It is also interesting to note that this choice of $s$ is the square root of the recently proposed "space-bandwidth ratio" [17].

The fact that the spatial bandwidth $\Delta \sigma_{x}^{\prime}$ of the field on the spherical reference surface decreases with increasing $z$ leads us to inquire whether the local bandwidth in the longitudinal direction also decreases with $z$. In other words, we may expect relatively more gradual changes in the field with increasing $z$, with respect to both the transverse and longitudinal dimensions, and thus use of a uniformly spaced grid with equal spacings between grid lines would not be representationally or computationally efficient. The purpose of this Letter is to show that this is indeed the case and quantitatively derive how the nonuniform grid spacings should be.

Our approach is based on approximating integrals involving chirps and is related to the stationary phase approximation [2]. The $\mp \infty$ limits of the Fresnel integral may be replaced by $\mp \Delta x / 2$, since $\hat{f}\left(x^{\prime}\right)$ is assumed zero outside this interval. The local frequency of the chirp function $\exp \left[i \pi\left(x-x^{\prime}\right)^{2} / \lambda z\right]$ inside the Fresnel integral is found by taking the derivative of its phase and dividing by $2 \pi$, yielding $-\left(x-x^{\prime}\right) / \lambda z$. Wherever the absolute value of this frequency exceeds the highest frequency of $\hat{f}\left(x^{\prime}\right)$, which is $\Delta \sigma_{x} / 2$, there will be negligible contribution to the integral, since the high-frequency chirp will wash out the signal. This will occur when $\left|\left(x^{\prime}-x\right) / \lambda z\right|>\Delta \sigma_{x} / 2$. Therefore, the lower integral limit need not be smaller than $\max \left(-\Delta x / 2, x-\Delta \sigma_{x} \lambda z / 2\right)$, and the upper integral limit need not be larger than $\min \left(\Delta x / 2, x+\Delta \sigma_{x} \lambda z / 2\right)$, unless these equations predict the lower limit to be greater than the upper limit, in which case the field at the point $(x, z)$ will be approximately zero. To proceed further, we will concentrate on the case $x=0$ for which the integral becomes symmetrical with the lower limit being the negative of the upper limit. Note that $\Delta \sigma_{x} \lambda z / 2$ will exceed $\Delta x / 2$ when $z>\Delta x / \Delta \sigma_{x} \lambda$, which precisely corresponds to the "knee of the curve" point discussed in [1]. Since to a good degree of approximation we can write $M=\sqrt{1+\lambda^{2} z^{2} / s^{4}} \approx \max \left(1, \lambda z / s^{2}\right)$ and $1 / M \approx \min \left(1, s^{2} / \lambda z\right)$ [18], the lower and upper integral limits can be compactly expressed as $\mp \Delta \sigma_{x} \lambda z / 2 M$ with $s^{2}=\Delta x / \Delta \sigma_{x}$.

The phase of the exponential in Fresnel's integral is $\pi x^{\prime 2} / \lambda z$. We will examine the change in this phase as a result of changes in $z$. We want to find the largest change in $z$ that will still not change the value of the Fresnel integral substantially. Assuming that $z$ changes by $\delta z$, the change in the phase is $\left[d\left(\pi x^{\prime 2} / \lambda z\right) / d z\right] \delta z=\left(-\pi x^{2} / \lambda z^{2}\right) \delta z$. This change in the phase will be largest when $x^{\prime}=$ $\mp \Delta \sigma_{x} \lambda z / 2 M$ and is equal to $-\pi \Delta \sigma_{x}^{2} \lambda \delta z / 4 M^{2}$. We equate this to $-2 \pi$, because we do not want the edge of the new chirp to deviate from that of the original chirp by more than a period, because a greater change would substantially affect the result of the Fresnel integral. This results in

$$
\delta z=\frac{8 M^{2}}{\lambda \Delta \sigma_{x}^{2}} .
$$

We note that $\delta z$ is an increasing function of $z$, since $M$ is an increasing function of $z$. This implies that the $z$ spacing will not be uniform in $z$; the spacing will increase with $z$. Now we will show that this nonuniform spacing with respect to $z$ corresponds to uniform spacing with respect to the FRT order parameter $a$. This suggests that increments in $a$ are more fundamental than increments in $z$ and affirms the intrinsic importance of the FRT order parameter in Fresnel propagation. The increment in $z$ is related to that in $a$ through $\delta z=(\partial z / \partial a) \delta a$. Using the expression for $M$ and $a$ from Eq. (3) to evaluate $\partial z / \partial a=\left(s^{2} / \lambda\right)(\pi / 2) \sec ^{2}(\pi a / 2)$, from Eq. (5) we obtain

$$
\delta a=\frac{16}{\pi} \frac{1}{\Delta x \Delta \sigma_{x}},
$$

where we have used $s^{2}=\Delta x / \Delta \sigma_{x}$. The $z$ independence of $\delta a$ implies uniform spacing with respect to $a$. Note that since $\Delta x \Delta \sigma_{x}=N$ is the space-bandwidth product of the original signal, the result is essentially $\delta a \sim 1 / N$. Since the nonredundant range of $a$ is of the order of unity, and in our case limited to [0,1], this means that there are $\sim N$ meaningfully distinguishable values of $a$ in question. That the number of meaningfully distinguishable values of $a$ turns out to be similar to the space-bandwidth product is a satisfying result.

An alternative of this approach is to work in the frequency domain, by calculating $\hat{g}(x, z)$ using the transfer function of free space:

$$
\hat{g}(x, z)=e^{i 2 \pi z / \lambda} \int_{-\infty}^{\infty} F\left(\sigma_{x}\right) e^{-i \pi \sigma_{x}^{2} \lambda z} e^{i 2 \pi \sigma_{x} x} \mathrm{~d} \sigma_{x} .
$$

Since $\hat{f}(x)$ is bandlimited, the limits of this integral will not be wider than from $-\Delta \sigma_{x} / 2$ to $\Delta \sigma_{x} / 2$. The local frequency of the chirp with respect to $\sigma_{x}$ is $-\left(\sigma_{x} \lambda z-x\right)$. Washout occurs when this frequency exceeds the maximum frequency in $F\left(\sigma_{x}\right)$, when $\left|\sigma_{x} \lambda z-x\right|>\Delta x / 2$. Thus the lower integral limit need not be smaller than $\max \left(-\Delta \sigma_{x} / 2,(-\Delta x / 2+x) / \lambda z\right)$, and the upper integral limit need not be larger than $\min \left(\Delta \sigma_{x} / 2,(\Delta x / 2+x) /\right.$ $\lambda z)$. Concentrating on the optical axis, we obtain the limits as a function of $z$. Note that the second terms above will dominate when $z>\Delta x / \Delta \sigma_{x} \lambda$. Again using $1 / M \approx$ $\min \left(1, s^{2} / \lambda z\right)$, the lower and upper limits can be compactly expressed as $\mp \Delta \sigma_{x} / 2 M=\mp \Delta \sigma_{x}^{\prime} / 2$ with $s^{2}=\Delta x / \Delta \sigma_{x}$. When $z$ changes by $\delta z$, the change in the phase of the chirp inside the integral is $\left[d\left(-\pi \sigma_{x}^{2} \lambda z\right) /\right.$ $d z] \delta z=-\pi \sigma_{x}^{2} \lambda \delta z$. This change in the phase will be largest when $\sigma_{x}=\mp \Delta \sigma_{x} / 2 M$ and is equal to $-\pi \Delta \sigma_{x}^{2} \lambda \delta z / 4 M^{2}$. The rest of the derivation leading to Eq. (6) follows as before.

An alternative approach will shed further light. We know that the integral in Eq. (7) needs to be evaluated 
only over a symmetrical interval of extent $\Delta \sigma_{x}^{\prime}=$ $\Delta \sigma_{x} / M$. While the symmetrical extent of $\hat{F}\left(\sigma_{x}\right)$ is originally specified as $\Delta \sigma_{x}$, we now observe that truncating its extent to $\Delta \sigma_{x}^{\prime}$ and using this truncated version in the integral will not change the resulting field. In other words, the frequency extent of $\hat{F}\left(\sigma_{x}\right)$ is effectively limited to $\Delta \sigma_{x}^{\prime}$. We refer to Eq. (1), which gives the 2D FT of $\hat{g}(x, z)$. Since we have seen that the effective frequency extent of $\hat{F}\left(\sigma_{x}\right)$ is $\Delta \sigma_{x}^{\prime}$, we observe that $\hat{G}\left(\sigma_{x}, \sigma_{z}\right)$ will be nonzero only between $1 / \lambda-\lambda\left(\Delta \sigma_{x}^{\prime} / 2\right)^{2} / 2$ and $1 / \lambda$. This is because the parabola will be truncated to zero for $\sigma_{z}<$ $1 / \lambda-\lambda \Delta \sigma_{x}^{\prime 2} / 8$ and the apex of the parabola is at $\sigma_{z}=1 / \lambda$ (Fig. 1). Thus the extent over which $\hat{G}\left(\sigma_{x}, \sigma_{z}\right)$ is nonzero along the $\sigma_{z}$ dimension is $\lambda \Delta \sigma_{x}^{\prime 2} / 8=\lambda \Delta \sigma_{x}^{2} / 8 M^{2}$. This $z$ bandwidth translates into $\delta z=8 M^{2} / \lambda \Delta \sigma_{x}^{2}$, consistent with our earlier Eq. (5). (Note that when $z=0$, we have $\Delta \sigma_{x}^{\prime}=\Delta \sigma_{x}$ and $M=1$. The $z$ bandwidth $\lambda \Delta \sigma_{x}^{2} / 8$ in this case is the global bandwidth of $\hat{g}(x, z)$ and will imply a $\delta z$ value of $8 / \lambda \Delta \sigma_{x}^{2}$. However, this $z$ independent spacing is a worst case result and does not account for the fact that the local $z$ bandwidth decreases with increasing $z$.)

We may also interpret these results more physically. The effective frequency extent $\Delta \sigma_{x}^{\prime}$ implies an angular divergence of $\lambda \Delta \sigma_{x}^{\prime}$. Over a distance of $\delta z$, this corresponds to a spread of $\lambda \Delta \sigma_{x}^{\prime} \delta z$. When this spread becomes comparable to the smallest feature of the transverse field profile, we will observe a substantial change in the transverse profile. Since the smallest feature size is $\sim 1 / \Delta \sigma_{x}^{\prime}$, we write $\lambda \Delta \sigma_{x}^{\prime} \delta z \sim 1 / \Delta \sigma_{x}^{\prime}$, which leads to $\delta z \sim 1 / \lambda \Delta \sigma_{x}^{\prime 2}=M^{2} / \lambda \Delta \sigma_{x}^{2}$, which is the same as Eq. (5) within numerical factors. In physical terms, the bandwidth and angular divergence decrease with increasing $z$. Smaller divergence means that there will be smaller changes in the wavefield for a given increment in $z$. Thus, we observe that variation of the field with respect to both the transverse and longitudinal dimensions becomes smaller with increasing $z$.

Finally, let us write $\hat{g}(x, z)=\hat{v}(x, z) \exp (i 2 \pi z / \lambda)$ and consider the paraxial Helmholtz equation: $\partial^{2} v(x, z) /$ $\partial x^{2}+(i 4 \pi / \lambda) \partial v(x, z) / \partial z=0$. Very crudely, we will define a substantial change as a change comparable to the value of the function itself: $|\partial v(x, z) / \partial z| \delta z \sim$ $|v(x, z)|$. Additionally, we would expect a signal to change substantially over a spatial extent that is comparable to the inverse of its frequency extent. Combining this with the previous idea applied to $x$, we get $|\partial v(x, z) / \partial x|\left(1 / \Delta \sigma_{x}^{\prime}\right) \sim|v(x, z)|$. The frequency extent of the derivative of a signal is the same as that of the signal itself. Thus writing the last result for $\partial v(x, z) / \partial x$ instead of $v(x, z)$ and using the last result to substitute for $\partial v(x, z) / \partial x$, we obtain $\left|\partial^{2} v(x, z) / \partial x^{2}\right| \sim \Delta \sigma_{x}^{\prime 2}|v(x, z)|$. Using these in Helmholtz's equation, we obtain $\Delta \sigma_{x}^{\prime 2}|v(x, z)| \sim(4 \pi / \lambda)(1 / \delta z)|v(x, z)|$, which again leads to Eq. (5) within numerical factors.

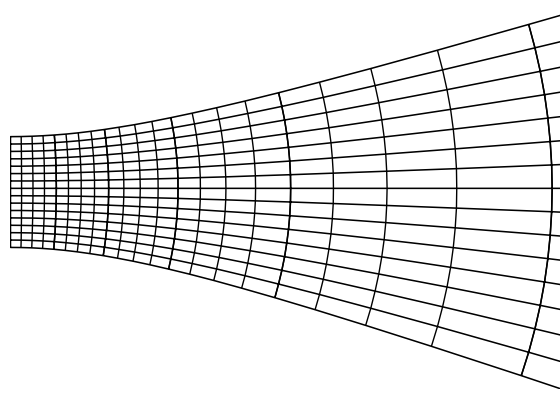

Fig. 2. Structure grid for Fresnel diffraction.

Figure 2 shows the grid implied by the $z$ dependent transverse spacing $1 / \Delta \sigma_{x}^{\prime}$ and longitudinal spacing $\delta z$. The consecutive spherical reference surfaces shown correspond to uniformly spaced FRT orders. This grid reflects the fundamental structure of Fresnel diffraction.

H. M. Ozaktas acknowledges the partial support of the Turkish Academy of Sciences.

\section{References}

1. H. M. Ozaktas, S. Ö. Arık, and T. Coşkun, Opt. Lett. 36, 2524 (2011).

2. H. M. Ozaktas, Z. Zalevsky, and M. A. Kutay, The Fractional Fourier Transform (Wiley, 2001).

3. H. M. Ozaktas and D. Mendlovic J. Opt. Soc. Am. A 12, 743 (1995).

4. H. M. Ozaktas and M. F. Erden, Opt. Commun. 143, 75 (1997)

5. H. M. Ozaktas, A. Koc, I. Sari, and M. A. Kutay, Opt. Lett. 31, 35 (2006).

6. A. Koc, H. M. Ozaktas, C. Candan, and M. A. Kutay, IEEE Trans. Signal Process. 56, 2383 (2008).

7. F. S. Oktem and H. M. Ozaktas, IEEE Signal Process. Lett. 16, 727 (2009).

8. F. S. Oktem and H. M. Ozaktas, J. Opt. Soc. Am. A 27, 1885 (2010).

9. L. Onural Appl. Opt. 39, 5929 (2000).

10. D. Mendlovic, Z. Zalevsky, and N. Konforti, J. Mod. Opt. 44, 407 (1997).

11. D. Mas, J. Garcia, C. Ferreira, L. M. Bernardo, and F. Marinho, Opt. Commun. 164, 233 (1999).

12. B. M. Hennelly and J. T. Sheridan, J. Opt. Soc. Am. A 22, 917 (2005).

13. B. M. Hennelly and J. T. Sheridan, J. Opt. Soc. Am. A 22, 928 (2005).

14. J. J. Healy, B. M. Hennelly, and J. T. Sheridan, Opt. Lett. 33, 2599 (2008).

15. J. J. Healy and J. T. Sheridan, J. Opt. Soc. Am. A 27, 21 (2010).

16. J. J. Healy and J. T. Sheridan, Opt. Lett. 35, 947 (2010).

17. J. J. Healy and J. T. Sheridan, J. Opt. Soc. Am. A 28, 786 (2011).

18. H. M. Ozaktas, Int. J. Elect. Eng. Edu. 31, 152 (1994). 